\title{
Structure and Mechanical Properties of Iron after Surface Severe Plastic Deformation under Friction with Simultaneous Nitrogen Saturation: I. Structure Formation
}

\author{
A. I. Yurkova ${ }^{a, *}$, Yu. V. Milman ${ }^{b}$, and A. V. Byakova ${ }^{a, b}$ \\ ${ }^{a}$ National Technical University of Ukraine Kiev Polytechnical Institute, Kyiv, 03056 Ukraine \\ ${ }^{b}$ Institute of Problems of Materials Science, National Academy of Sciences of Ukraine, Kyiv, 03680 Ukraine \\ *e-mail:yurkova@kpm.ntu-kpi.kiev.ua
}

\begin{abstract}
The structure of armco iron after severe plastic deformation under friction with simultaneous nitrogen saturation has been studied by microstructure analysis, X-ray diffraction, and transmission and scanning electron microscopy. A gradient surface layer, in which the grain size varies from micro- to submicro- and nanometer-scale values, is found to form. A mutual intensifying effect of the deformation-induced refinement of a grain structure and the diffusion of nitrogen atoms in iron is shown. The formation of a nanocrystalline state is discussed as a result of dynamic recrystallization.
\end{abstract}

DOI: $10.1134 / \mathrm{S} 0036029512040143$

\section{INTRODUCTION}

This work is a continuation of the studies of the iron structure obtained by surface severe plastic deformation under friction (SPDF) in a neutral argon atmosphere [1]. Based on the experimental data, we showed that a nanocrystalline iron structure can form during SPDF under conditions of dynamic recrystallization during hot deformation, which is provided deformation at different directions, a rate above $10^{2} \mathrm{~s}^{-1}$, and a temperature of $773 \mathrm{~K}$.

Interest to studying the structure and properties of materials formed under high-energy mechanical actions by severe plastic deformation (SPD) that lead to the refinement of a grain structure to submicro- and nanosizes is caused by the possibility of obtaining a high level of physicochemical and mechanical properties and, therefore, high operating characteristics of materials [2-8].

From an analysis of the experimental and theoretical results presented in [2-6, 9], it follows that the structural peculiarities and properties of nanomaterials are substantially dependent on their compositions and the method of preparing. A high-energy mechanical action can create optimal conditions for physicochemical processes for the most effective energy and material exchange both in the material under processing and in the environment.

The conditions of producing nanomaterials by SPD provide the formation of a nonequilibrium state with a fine-grained structure. It is known that such structures are characterized by an anomalous diffusion mass transfer and that the solubility of the components increases significantly [4, 9-12]. Unlike the methods of bulk deformation $[2-4,6]$, SPD methods allow one to change the phase composition of the surface layer of a material during structure formation due to the diffusion of interstitial atoms from an external medium. Despite this fact, in world practices, the problem of refining a grain structure during SPD with simultaneous saturation with interstitial atoms is scantly known, although its solution has great importance for an effective control of chemical and phase compositions, retarding the processes of recovery and recrystallization, and an increase in the tendency of a material toward grain refinement in the zone of influence of SPD and the conservation of the obtained ultrafine-grained structure.

One of the effective methods of grain structure refinement to nanosizes can be a combination of highenergy mechanical actions, e.g., surface SPDF with simultaneous nitrogen saturation [10]. A practical application of such treatment is retarded by the lack of data on the phase and structural changes occurring under these conditions.

In the specific characteristics, friction is one of the most power sources of a high-energy action on materials, as a process of fast supply of mechanical energy. Therefore, an attempt to obtain ultrafine-grained surface layers with qualitatively new physicochemical and mechanical properties using friction is thought to be justified.

The aim of this work is to study the structural changes in iron under conditions of SPDF with simultaneous nitrogen saturation.

\section{EXPERIMENTAL}

In this work, as in [1], we studied iron, which is a basis of structural alloys most widely used in engineer- 
ing. It is known that nitriding is also widely used to increase the mechanical properties of iron and alloys. In this work, the results of nitriding during SPDF are compared to the results of conventional furnace nitriding at the same temperature.

The studies were performed on cylindrical samples ( $8 \mathrm{~mm}$ in diameter and $50 \mathrm{~mm}$ in height) of an armco iron of 99.97 wt \% purity. Annealed samples with a coarse-grained structure (grain sizes $80-100 \mu \mathrm{m}$ ) were subjected to SPDF in the special-purpose setup described in [1] in an ammonia atmosphere at a constant deformation temperature of $773 \mathrm{~K}$ for $1 \mathrm{~h}$. (The results of preliminary studies showed that it is the temperature at which a nanocrystalline structure forms in the surface layer under the experimental conditions).

The parameters of treatment (temperature, time, and strain rate) were chosen the same as during SPDF of iron in a neutral argon atmosphere [1]; the parameters provide dynamic recrystallization conditions. The samples were heated to the given temperature $(773 \mathrm{~K})$ due to the friction energy released during the rotation of a sample at a rate of $6000 \mathrm{rpm}$ between hard alloy VK8 plates forced against the sample surface. Furnace nitriding was carried out at a temperature of $773 \mathrm{~K}$ in an ammonia atmosphere.

The structure and phase composition of the samples after SPDF in an ammonia atmosphere were studied using the methods as follows: optical microscopy (Netphot-21 microscope, resolution of $0.4 \mu \mathrm{m}$ ), $\mathrm{X}$-ray diffraction (DRON-4.13 diffractometer, $20 \mathrm{kV}$, $10 \mathrm{~mA}$ ) using the characteristic radiation of an iron anode and also by photographing a thin foil by transmitting monochromatized (monochromator is a bent quartz crystal) radiation of an iron anode in a Guinier chamber of the Enraf Nonius Delft FR-503G type with the Zeeman-Bolin focusing, scanning electron microscopy (FZJ-IWV2004 microscope, operating voltage $\mathrm{EHT}=10 \mathrm{kV}$, working distance $\mathrm{WD}=11 \mathrm{~mm}$ ) by the method of electron backscattering diffraction (EBSD) using an orientation system of images, and transmission electron microscopy (JTM-CX microscope, accelerating voltage $125 \mathrm{kV}$ ). The study of the structure with the JEM-CX microscope was performed under conditions of bright-field and dark-field images and microdiffraction. The grain size was found from a dark-field image as the average grain diameter.

\section{EXPERIMENTAL RESULTS}

It was found in [1], where samples were studied after SPDF in an argon atmosphere, that a characteristic peculiarity of the deformed surface layers is a structural gradient, namely, a decrease in the grain sizes from micro- to submicro- and nanometer-scale level and an increase in the dislocation density in the direction from the undeformed base to the surface. In this case, the average dislocation density increases by almost five orders of magnitude from $10^{11} \mathrm{~m}^{-2}$ in the undeformed base to $10^{16} \mathrm{~m}^{-2}$ at the surface; the average coherent domain size at the surface is $13 \mathrm{~nm}$. According to the electron microscopy data, the grain size in the surface layer is approximately $20 \mathrm{~nm}$. Based on the experimental data, it was shown that a nanocrystalline iron structure can be formed during SPDF as a result of dynamic recrystallization during hot deformation that is provided by variously directed deformation at a rate higher than $10^{2} \mathrm{~s}^{-1}$.

In this work, the SPDF conditions were the same as in [1], which allowed us to clearly characterize the influence of nitrogen saturation simultaneous with SPDF on the structure and properties of the surface layers. During SPDF of iron in an ammonia atmosphere, as well as under friction in argon atmosphere, a gradient layer with different degrees of dispersion of a grain structure forms at the sample surface (Fig. 1a): from micro- (regions 4 and 3) to submicro- and nanometer-size (regions 2 and 1 ) dispersion. However, the thicknesses of the entire dispersed layer and each structural region are significantly larger than those after SPDF in an argon atmosphere.

The usual methods of optical microscopy do not detect grain boundaries in the regions adjacent to the surface (regions 1,2), and these methods detect highdisperse grains in the treatment zone in the material only at some distance from the surface (region 3). The microstructure of region 4 of the deformed layer is characterized by a pronounced grain shape anisotropy (morphological texture), and it consists of elongated structural elements disposed at an angle to the sample radius. This angle $(\beta)$ decreases with increasing distance from the surface.

The electron microscopy studies confirm a gradient character of iron after SPDF. In layer regions with tilted elongated and equiaxed microsized grains (regions 4, 3), a cellular structure forms. As the surface is approached and the rate and degree of deformation increase, the grains of the initial structure are fragmented into submicrometer- (Fig. 1b) and nanometer-scale (Figs. 1c, 1d) grains. In the surface layer, the grain size determined from a dark-field image is about $10 \mathrm{~nm}$ (Fig. 1d).

The electron microdiffraction pattern (Fig. 1c) obtained from a foil portion with an area of about $0.25 \mu \mathrm{m}^{2}$ contains very diffuse interference rings consisting of numerous point reflections. The existence of individual reflections arranged on a ring indicates the formation of a granular structure with high-angle grain boundaries $[2,3,14-16]$. The significant smearing of the diffraction pattern indicates a high dispersion of the grain structure, a substantial level of microstresses, a high chemical inhomogeneity, and a crystal lattice distortion related to a high nitrogen content in $\alpha-\mathrm{Fe}$. All reflections in the electron diffraction patterns belong to the $\alpha-\mathrm{Fe}(\mathrm{N})$ crystal lattice, i.e., the solid solution of nitrogen in iron; no other phases were detected. 


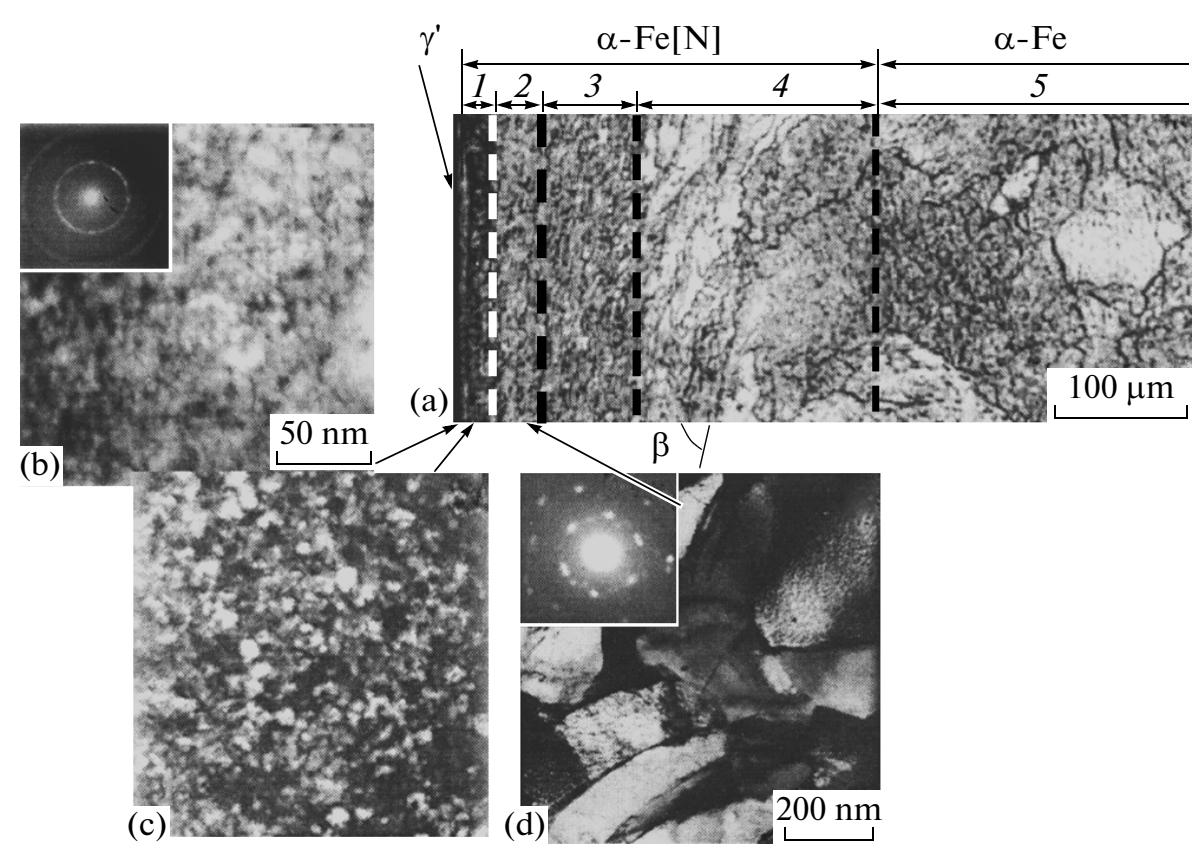

Fig. 1. Structure of armco iron after SPDF with simultaneous nitrogen saturation. (a) $\mathrm{Fe}_{4} \mathrm{~N}$ ( $\gamma^{\prime}$ phase) layer and $\alpha-\mathrm{Fe}[\mathrm{N}]$ disperse layer regions: (1) nanocrystalline, (2) submicron grains, (3) microcrystalline with equiaxed grains, (4) microcrystalline with morphological texture, and (5) base metal. (b), (c) Bright-field image and microdiffraction pattern of the disperse layer regions shown by the arrows in (a) (electron microscopy). (d) Dark-field image taken with the (110) $\alpha$ reflection.

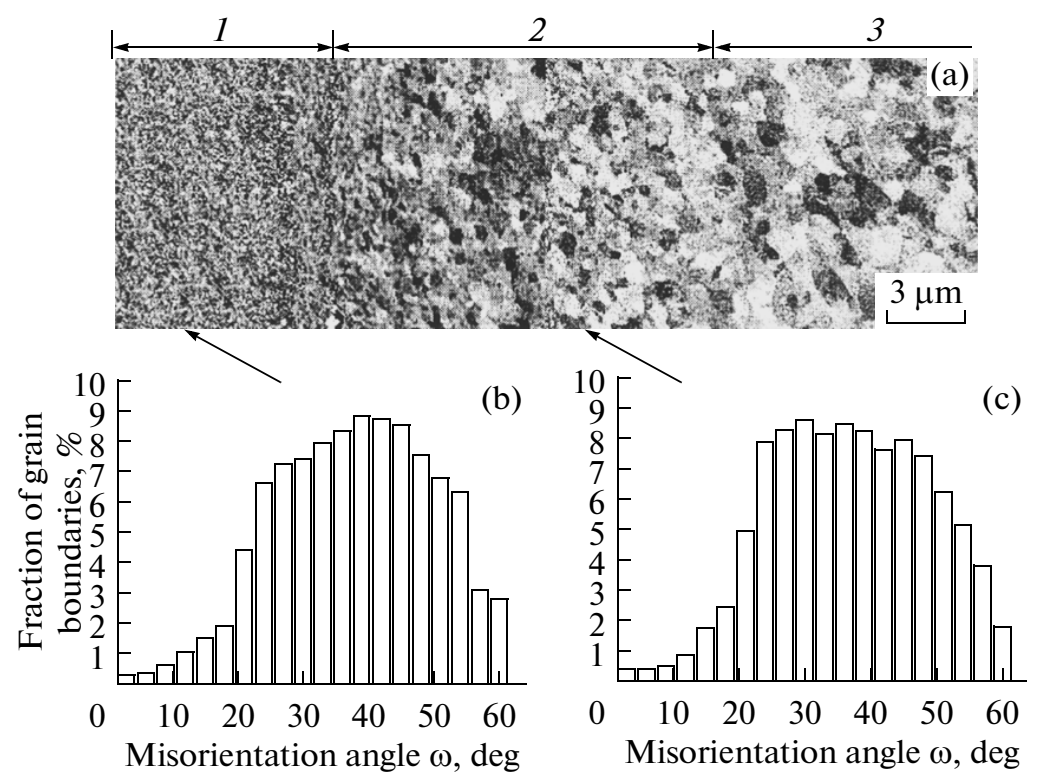

Fig. 2. (a) Structure of armco iron after SPDF with simultaneous nitrogen saturation and (b), (c) misorientation angle distribution of grain boundaries: (1) nanocrystalline structure, (2) submicrocrystalline structure, (3) microcrystalline structure with equiaxed grains.

The formation of a grain-type structure with highangle boundaries is confirmed by different contrast of neighboring crystallites (Fig. 2a) obtained by electron backscattering diffraction (EBSD) method [17]; this contrast indicates different crystallographic orientations of the grains. The misorientation distribution of crystallite boundaries established by EBSD using an orientation system of images shows that they are predominantly high-angle, as in the case after SPDF in a neutral atmosphere (argon).

X-ray diffraction (XRD) shows that SPDF with simultaneous nitrogen saturation causes the formation 


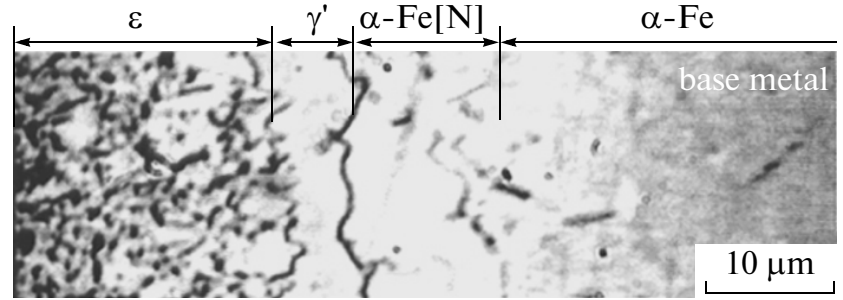

Fig. 3. Microstructure of iron after furnace nitriding at $773 \mathrm{~K}$ for $2 \mathrm{~h}$.

of a thin (less than $3 \mu \mathrm{m}$ ) layer of cubic nitride $\mathrm{Fe}_{4} \mathrm{~N}$ ( $\gamma^{\prime}$-phase), which is well revelaed upon metallographic studies (Fig. 1). Hexagonal nitride $\mathrm{Fe}_{[2-3]} \mathrm{N}$ ( $\varepsilon$-phase), a thick layer of which always forms at the iron surface under conditions of nitrogen saturation without deformation (upon furnace nitriding) and subsequent cooling [18] (Fig. 3], is almost absent after SPDF in an ammonia atmosphere. $\mathrm{X}$-ray diffraction patterns show only the traces of $\mathrm{Fe}_{2-3} \mathrm{~N}$ at the sample surface, and metallographic studies do not detect this nitride.

Layer-by-layer XRD of the samples after SPDF with simultaneous nitrogen saturation shows that the $\alpha-\mathrm{Fe}[\mathrm{N}]$ phase is the basis of regions $1-4$ in the surface layer (Fig. 1) and there are no precipitates of nitride phases there. The X-ray diffraction patterns of the samples taken with monochromatic radiation, which provides a higher sensitivity to phases, do not detect any precipitates of iron nitrides or other phases in regions $1-4$ of the layer saturated with nitrogen during SPDF in any marked amounts: this layer completely consists of nitrogen ferrite (Fig. 4). The results are confirmed by transmission electron microscopy (TEM) data. The interferences of the $\alpha-\mathrm{Fe}(\mathrm{N})$ solid solution have strong smearing, and they are significantly shifted toward lower Bragg angles as compared
Fig. 4. (110) Interference line of the $\alpha-\mathrm{Fe}[\mathrm{N}]$ phase after SPDF with simultaneous nitrogen saturation.

to the initial annealed state (Table 1). The changes in the lattice parameter and nitrogen content in the $\alpha-\mathrm{Fe}[\mathrm{N}]$ phase across the thickness of the layer subjected to SPDF with simultaneous nitrogen saturation, which were determined from the position of the (220) line of the $\alpha$ phase according to the procedure described in [19], are shown in Table 1 and Fig. 5.

The nitrogen saturation during SPDF in an ammonia atmosphere favors higher refining of the grain structure as compared to friction in an argon atmosphere [1]. It is found that the dispersion of the grain structure very strong influences the diffusion activity and solubility of nitrogen in $\alpha-\mathrm{Fe}$ during deformation under friction. The thickness of the layer saturated with nitrogen during SPDF is much more than the thickness of the nitrided layer obtained during nitrogen saturation with no deformation [18] (Fig. 5), and the nitrogen content in the refined $\alpha-\mathrm{Fe}(\mathrm{N})$ solid solution (in a $100-\mu \mathrm{m}$-thick layer) is higher by a factor of three as compared to the nitrogen concentration in $\alpha-\mathrm{Fe}$ that is determined by the $\mathrm{Fe}-\mathrm{N}$ equilibrium diagram at the temperature of its maximum solubility $\left(863 \mathrm{~K}\left(590^{\circ} \mathrm{C}\right)\right)$ and is two order of magnitude higher than the usual nitrogen content in coarse-grained $\alpha-\mathrm{Fe}(\mathrm{N})$ after furnace nitriding at $773-863 \mathrm{~K}$ with no deformation and after cooling to room temperature [18].

The strong smearing and shift of the (220) interference lines of $\alpha-\mathrm{Fe}[\mathrm{N}]$ are caused by its structural state and are due to a high dislocation density, structure dispersion, and a high solubility of interstitial elements

Table 1. Changes in the profile shape and position of the (220) $K_{\alpha}$ diffraction line, the lattice parameter, and the nitrogen concentration across the surface layer thickness in armco iron after SPDF with simultaneous nitrogen saturation

\begin{tabular}{|c|c|c|c|}
\hline $\begin{array}{l}\text { Distance from the surface, } \\
\qquad \mu \mathrm{m}\end{array}$ & (220) $K_{\alpha}$ line profile & Lattice parameter, nm & Nitrogen content, wt \% \\
\hline 5 & & 0.2872 & 0.31 \\
\hline 30 & & 0.2872 & 0.31 \\
\hline 50 & & 0.2872 & 0.31 \\
\hline 100 & & 0.2872 & 0.31 \\
\hline 150 & & 0.2871 & 0.24 \\
\hline 200 & & 0.287 & 0.14 \\
\hline 250 & & 0.2867 & 0.003 \\
\hline 350 & 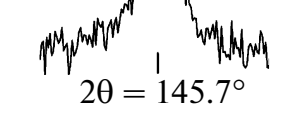 & 0.2867 & 0.003 \\
\hline
\end{tabular}




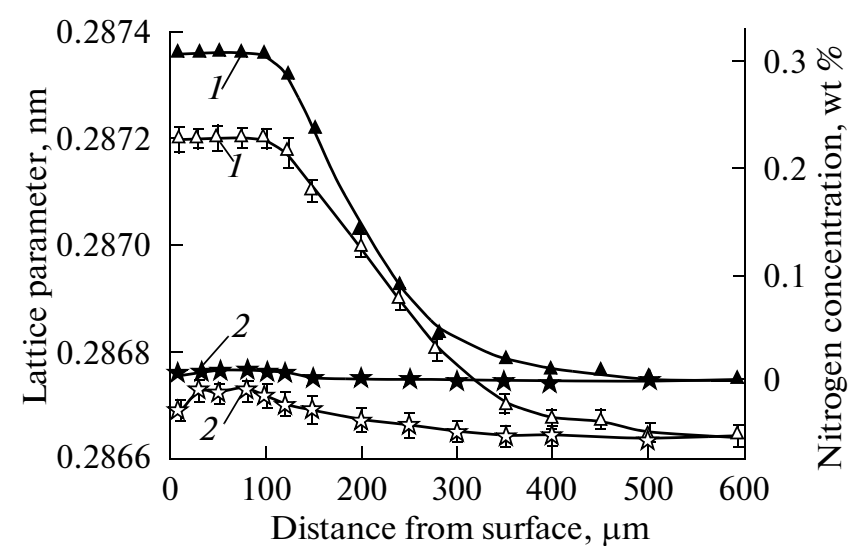

Fig. 5. (open symbols) $\alpha-\mathrm{Fe}$ lattice parameter and (solid symbols) nitrogen concentration in $\alpha$-Fe vs. the surface layer thickness after (1) nitrogen saturation at $773 \mathrm{~K}$ during SPGF and (2) furnace nitriding.

within $\alpha-\mathrm{Fe}$ nanograin boundaries [11]. A high nitrogen solubility in the $\alpha$ phase determines the absence of precipitates of nitride phases in the dispersed layer.

Using layer-by-layer XRD and hardness measurements (as will be shown in part II of this paper), we determined the diffusion layer thickness after SPDF in an ammonia atmosphere and after furnace nitriding upon holdings for $0.5,1,1.5,2$, and $3 \mathrm{~h}$ and constructed kinetic curves for the diffusion layer growth (Fig. 6). Based on the results of many experimental studies [20-22], we used the initial law of diffusion layer growth in the form of the power dependence

$$
h^{n}=k \tau,
$$

where $h$ is the diffusion layer thickness, $\tau$ is the isothermal holding time, and $k$ is a coefficient.

Coefficient $k$ and exponent $n$ were found by constructing the graphic dependence in the $\ln h-\ln \tau$ coordinates. As a result, we obtained $n=2$ and $k=7.84 \times 10^{-9}$ for furnace nitriding and $n=1.82$ and $k=5.13 \times 10^{-7}$ for SPDF with simultaneous nitrogen diffusion. Exponent $n=2$ found for the case of steady heating during furnace nitriding agrees well with the generally accepted concepts that an increase in diffusion layer thickness $h$ with time $\tau$ obeys the parabolic law $h_{2}=k \tau$ (or $h=\sqrt{k \tau}$ ) [20-23]; therefore, coefficient $k$ is diffusion coefficient $D$ of nitrogen in iron. In this case, the value $k=7.84 \times 10^{-9}$ agrees well with the values of the diffusion coefficient of nitrogen in $\alpha-\mathrm{Fe}$ at $773 \mathrm{~K}(D=$ $\left.(3.93-13.8) \times 10^{-9} \mathrm{~cm}^{2} / \mathrm{s}\right)$ calculated from the temperature dependences in [24]. In the case of SPDF with simultaneous nitrogen saturation, exponent $n=$ 1.82 differs from the exponent for the purely diffusion process, and the value of $k$ is approximately two orders of magnitude higher. In this case, coefficient $k$ has no adequate physical meaning and, according to [25], is a

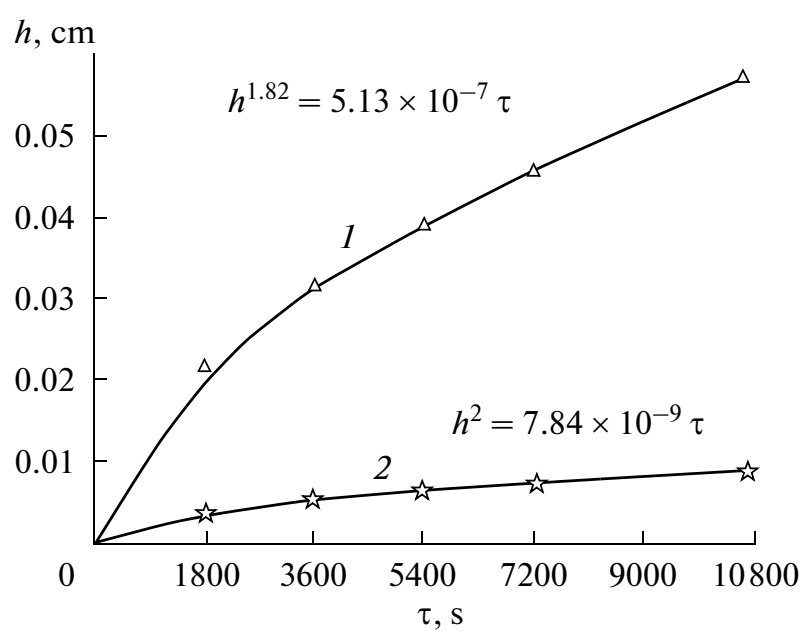

Fig. 6. Kinetic curves for the diffusion layer growth in armco iron measured after (1) SPDF nitrogen saturation at $773 \mathrm{~K}$ and (2) furnace nitriding.

"practical" constant of the process rate. Coefficient $k$ can also be called the effective diffusion coefficient during SPDF. The deviation of the law of layer growth from the parabolic law is likely due to the influence of plastic deformation during SPDF nitrogen saturation. In this case, nitrogen diffusion is accelerated due to diffusion along grain and subgrain boundaries and also along dislocation lines. The mass transfer under plastic deformation conditions has specific features and can occur by a mechanism other than a usual diffusion mechanism [26].

An estimation of the SPDF contribution to the acceleration of nitrogen diffusion shows that the time of formation of a $100-\mu \mathrm{m}$-thick nitrided layer on iron during deformation under friction decreases by almost an order of value as compared to the nitrogen saturation with no deformation.

After SPDF of iron in an argon atmosphere, the degree of true strain $e$ at the sample surface found in [1] was 13.5 , and the corresponding shear strain was $\gamma=23.5$. Since the SPDF conditions (the temperature, the rate of sample rotation, and the treatment time) and the hardness of the surface nanocrystalline layer prepared in both ammonia and argon atmospheres (part II of this work) are the same, the degrees of deformation of a thin surface layer during SPDF in ammonia and argon atmospheres are assumed to be the same.

The results show that the thicknesses of the entire deformation layer and each of its structural regions after friction in ammonia aree larger than after friction in argon.

\section{DISCUSSION OF THE RESULTS}

The deformation fragmentation of a grain structure under SPDF conditions (treatment in the temperature 

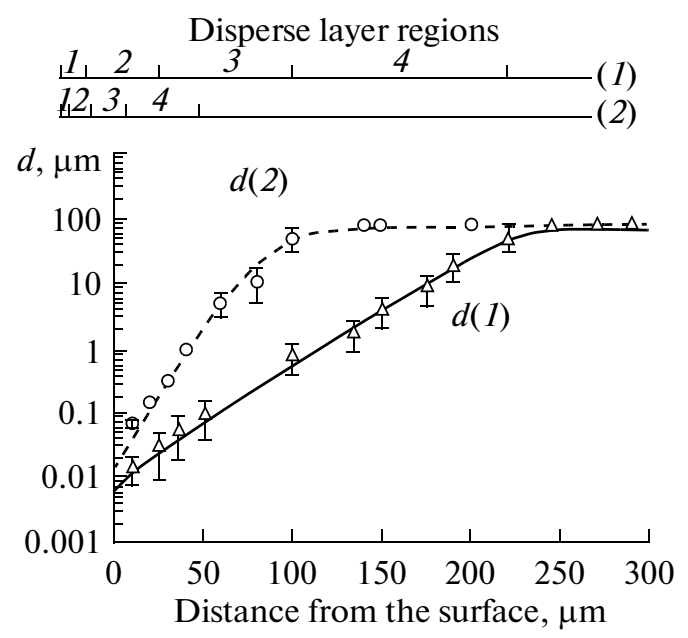

Fig. 7. Changes in grain size $d$ across the surface layer thickness in armco iron after SPDF in (1) ammonia and (2) argon [1]. Disperse layer regions: (1) nanocrystalline, (2) submicron grains, (3) microcrystalline with equiaxed grains, (4) microcrystalline with a morphological texture.

range of hot deformation using the mechanism of dynamic recrystallization that is provided by variously directed deformation at a high rate $\bar{\varepsilon}>10^{2} \mathrm{~s}^{-1}$ at $773 \mathrm{~K}$ ) [1] is due to formation, motion, redistribution of structural defects and to recrystallization in the most strongly distortion portions. The surface layer formed during SPDF is in an active state, which substantially increases the reactivity and diffusion susceptibility of the material during its treatment in active media. Atomic nitrogen (the product of ammonia dissociation on the sample surface heated by friction) interacts actively with iron. In the case of heating of iron with no deformation, nitrogen reacts with iron, forming nitrogen solid solution $(\alpha-\mathrm{Fe}[\mathrm{N}])$ and metastable nitrides ( $\gamma^{\prime}$ and $\varepsilon$ phases) $[18,23,24]$.

A high diffusion rate of nitrogen atoms substantially influences the refining of an iron grain structure during SPDF; the influence is expressed as an increase in the disperse layer thickness and the degree of refining upon SPDF in an active nitrogen-containing atmosphere as compared to SPDF in argon atmosphere (Fig. 7, Table 2). After SPDF with simultaneous nitrogen saturation, the disperse layer thickness is larger by a factor of 3.5 and the minimum grain size in the nanocrystalline region of the $\alpha-\mathrm{Fe}[\mathrm{N}]$ solid solution adjacent to the $\gamma^{\prime}$ phase layer is half that in $\alpha$-Fe after SPDF in argon.

According to [28, 29], grain size $d$ during dynamic recrystallization is determined by the semiempirical relationship

$$
d=k Z^{-m}
$$

Here, $d$ is the grain size $(\mu \mathrm{m}) ; Z=\bar{\varepsilon} \exp \frac{Q}{R T}$ is the Zener-Hollomon parameter taking into account the

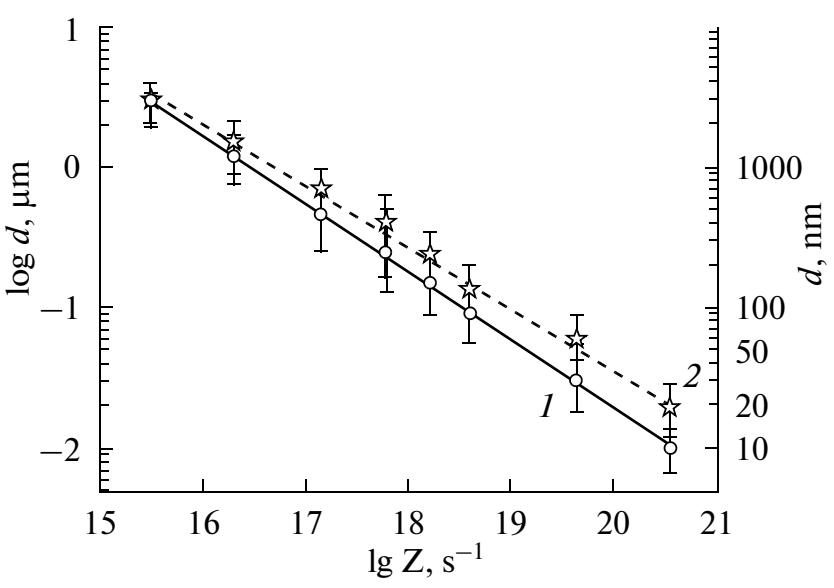

Fig. 8. Grain size $d$ vs. Zener-Hollomon parameter $Z$ after (1) SPDF with simultaneous nitrogen saturation and (2) SPDF in argon.

influence of the deformation temperature and rate, where $\bar{\varepsilon}$ is the strain rate $\left(\mathrm{s}^{-1}\right) ; T$ is the deformation temperature; $Q$ is the activation energy of grainboundary migration $(254 \mathrm{~kJ} / \mathrm{mol})$, which is close to the self-diffusion activation energy of iron atoms; and $R$ is the gas constant.

To obtain a fine grain, parameter $Z$ should be increased, which is provided by an increase in strain rate $\bar{\varepsilon}$ at a constant temperature [1].

Since the strains in similar structural regions of the iron surface layer upon SPDF in argon and ammonia are almost the same (without regard for the deformation because of lattice distortions induced by dissolved nitrogen atoms) (Fig.7), it should be suggested that the strain rates are almost the same as well. On this basis, the strain rate at the sample surface during SPDF with simultaneous nitrogen diffusion is $\bar{\varepsilon}_{\max }=2.4 \times 10^{3} \mathrm{~s}^{-1}$ and the Zener-Hollomon parameter is $Z=3.6 \times 10^{20} \mathrm{~s}^{-1}$ at a constant temperature $T=773 \mathrm{~K}$; i.e., they are the same as in the case of SPDF in argon [1]. In this case, the minimum grain size after SPGF in ammonia is half that after SPDF in argon.

With allowance for the equality of the strain rates during SPDF in argon and ammonia, we constructed dependences (2) in logarithmic coordinates (Fig. 8). The processing of the results gives $m=0.49$ and $k=$ $11.7 \times 10^{7}$ for SPDF with simultaneous nitrogen diffusion and $m=0.43$ and $k=1.48 \times 10^{7}$ for SPDF in argon

Table 2. Parameters of the surface layer in armco-iron after SPDF in gaseous media

\begin{tabular}{l|l|c|c}
\hline \multirow{2}{*}{ Medium } & \multicolumn{2}{|c|}{ Layer thickness, $\mu \mathrm{m}$} & \multirow{2}{*}{$\begin{array}{c}\text { Minimum grain } \\
\text { size, } \mathrm{nm}\end{array}$} \\
\cline { 2 - 3 } & dispersed & deformed & \\
\hline Argon & $40-50$ & 80 & 20 \\
Ammonia & $16-170$ & 300 & 10 \\
\hline
\end{tabular}


[1]. In the grain size range under study $(d<2 \mu \mathrm{m})$, SPDF with simultaneous nitrogen diffusion leads to grain refinement (see Fig. 8): the smaller the grain size, the stronger the effect. It may be suggested that the nitrogen influence disappears at larger grain sizes.

In addition, we have revealed a significant influence of a dispersed grain structure on the diffusion activity and high nitrogen solubility in $\alpha-F e$ during SPDF. The dislocation density in the dispersed layer saturated with nitrogen cannot be measured, since a high nitrogen concentration substantially influences the diffraction line width. However, taking into account that nitrogen atoms retard dislocation motion, we suggest that the dislocation density after SPDF in ammonia is higher than that after SPDF in argon at the same grain size. As a result, the rate of nucleation of recrystallization centers increases, whereas the migration rate of grain boundaries decreases by nitrogen atoms segregating on them. The increase in the number of recrystallization centers with decreasing migration rate of grain boundaries is likely to result in grain refinement under dynamic recrystallization conditions during SPDF in ammonia.

Thus, the main difference between SPDF with simultaneous nitrogen saturation and SPDF in a neutral medium is the mutual intensifying influence of deformation-induced grain refinement and the mass transfer of nitrogen atoms in iron: they intensify structural evolution during deformation and enhance the nitriding efficiency. This is the reason why SPDF with simultaneous nitrogen saturation significantly increases the dispersed-structure layer thickness, decreases the minimum grain size, increases the nitrided layer thickness, and provides an anomalously high nitrogen content in the layer.

\section{CONCLUSIONS}

(1) SPD with simultaneous nitrogen saturation under dynamic recrystallization conditions (temperature of $773 \mathrm{~K}$, variously directed deformation rate $\bar{\varepsilon}>$ $10^{2} \mathrm{~s}^{-1}$ ) leads to the formation of a gradient structure with grain sizes decreasing from initial $80-100 \mu \mathrm{m}$ to nanocrystalline $10 \mathrm{~nm}$ at the iron sample surface. In this case, the nanograin size at the surface is smaller by a factor of two and the thickness of a nanocrystalline region in the alloy is larger by a factor of three as compared to SPDF in a neutral medium.

(2) As compared to furnace nitriding at the same temperature $(773 \mathrm{~K})$, the diffusion rate of nitrogen atoms increases by almost two orders of magnitude, the thickness of the nitrided layer formed at the same time increases by a factor of five-six, and the nitrogen concentration in the nitrided layer measured at room temperature increases by two orders of magnitude.

(3) The decrease in the nanograin size during SPDF with simultaneous nitrogen saturation under dynamic recrystallization conditions is associated with a decrease in the dislocation mobility in the presence of nitrogen atoms, which increases the dislocation density and the nucleation rate of recrystallization centers and decreases the migration rate of grain boundaries.

\section{ACKNOWLEDGMENTS}

This work was supported in part by the Ministry of Education and Science of Ukraine (project no. 2211-f) and the National Academy of Sciences of Ukraine (project no. III-14-07).

\section{REFERENCES}

1. A. I. Yurkova, Yu. V. Milman, and A. V. Byakova, 1 "Structure and the Mechanical Properties of Iron after Surface Severe Plastic Deformation under Friction: I. Specific Features of Formation of the Structure," Deform. Razrushenie Mater., No. 1, 2-11 (2009).

2. N. I. Noskova and P. P. Mulyukov, Submicrocrystalline and Nanocrystalline Metals and Alloys (UrO RAN, Yekaterinburg, 2003).

3. R. Z. Valiev and I. V. Aleksandrov, Nanostructured Materials Produced by Severe Plastic Deformation (Logos, Moscow, 2000).

4. Yu. R. Kolobov, R. Z. Valiev, G. P. Grabovetskaya, et al., Grain-Boundary Diffusion and the Properties of Nanostructured Materials (Nauka, Novosibirsk, 2001).

5. P. A. Andrievskii and A. M. Glezer, "Size Effects in Nanocrystalline Materials: 1. Structure Characteristics, Thermodynamics, Phase Equilibriums, and Transport Phenomena (Phys. Met. Metallogr. 88 (1), 45-66 (1999)); 2. Mechanical and Physical Properties," Phys. Met. Metallogr. 89 (1) 83-103 (2000).

6. R. Z. Valiev and M. Yu. Murashkin, "Bulk Nanostructured Materials: Methods of Fabricating, Unusual Properties and Perspectives of Application," in Structural Materials and Quality Control Methods, Ed. By D. L. Meerson (TGU-MISiS, Tula-Moscow, 2007), pp. 111-170.

7. V. V. Rybin, "Regularities of Formation of Mesostructures during Developed Plastic Deformation," Vopr. Metalloved. 29 (1), 11-33 (2002).

8. S. Firstov, M. Brodnikovsky, M. Danylrnko, and Yu. Podrezov, "Nanocrystalline Structure Formation under Severe Plastic Deformation and Its Influence on Mechanical Properties," Rev. Adv. Mater. Sci. 4, 155162 (2003).

9. A. P. Shpak, Yu. A. Kunitskii, and V. L. Karbovskii, Cluster and Nanostructured Materials, Vol. 1 (Akademperiodika, Kiev, 2001).

10. A. Yurkova, A. Belots'ky, and A. Byakova, "Anomalous 1 Nitrogen Solubility in Gradient Nanostructured Layer Formed in the Surface of Bulk Iron by Severe Plastic Deformation under Friction," in Nanostructured Materials by High-Pressure Severe Plastic Deformation (Springer, Netherlands, 2006), Vol. 212, pp. 107-112.

11. Yu. V. Milman, K. E. Grinkevich, S. I. Chuganova, et al., "Tribological Properties of the Surface of Railway 
Tracks Studied by Indentation Technique,” Wear 258, 77-82 (2005).

12. V. V. Gorskii, V. V. Tikhonovich, and B. S. Shapoval, "Structure Changes in Surface Layers of a 130Kh16M Steel-20Kh13 Steel Friction Pair," Metallofizika 6 (11), 56-62 (1985).

13. M. Umemoto, Y. Todaka, J. Li, and K. Tsuchiya, "Nanocrystalline Structure in Steel Produced by Various Severe Plastic Deformation Processes," Mater. Sci. Forum 503-504, 11-18 (2006).

14. N. R. Tao, Z. N. Wang, W. P, Tong, et al., "An Investigation of Surface Nanocrystallization Mechanism in Fe Induced by Surface Mechanical Attriction Treatment," Acta Mater. 50, 4603-4616 (2002).

15. Yu. V. Ivanisenko, A. V. Korznikov, I. M. Safarov, et al., "Formation of Ultrafine-Dispersed Structure in Iron and Its Alloys upon Severe Plastic Deformations," Izv. Ross. Akad. Nauk, Ser. Met., No. 6, 126-131 (1995).

16. T. I. Chashchukhina, M. V. Degtyarev, L. M. Voronova, L. S. Davydova, and V. P. Pilyugin, "Effect of the Deformation Mode on the Hardness and Structure of Armco Iron and Structural Steel upon Deformation and Subsequent Annealing,” Phys. Met. Metallogr. 91 (5), 500-508 (2001).

17. H. P. Stüuwe, "Crystal Restoration during Severe Plastic Deformation," Mater. Sci. Forum 503-504, 175178 (2006).

18. R. Chatterjee-Fischer, F.-W. Eysell, R. Hoffmann, et al. Nitriding and Carbonitriding (Gasgemishen, VDIVerlag GmbH, Düsseldorf, 1985).
19. A. V. Belotskii, "Structure of Nitrogen Phases and Principles of Steel Alloying for Nitriding," Metalloved. Term. Obrab. Met., No. 12, 24-27 (1975).

20. B. S. Bokshtein, S. Z. Bokshtein, and A. A. Zhukhovitskii, Thermodynamics and Kinetics of Diffusion in Solids (Metallurgiya, Moscow, 1974).

21. S. Z. Bokshtein, Diffusion in Metals (Metallurgiya, Moscow, 1978).

22. L. N. Larikov and V. I. Isaichev, Diffusion in Metals and Alloys, Handbook (Naukova Dumka, Kiev, 1987).

23. Yu. M. Lakhtin and Ya. D. Kogan, Nitriding of Steel (Mashinostroenie, Moscow, 1976).

24. Yu. M. Lakhtin, Ya. D. Kogan, G. Shpis, and Z. Bemer, Theory and Technology of Nitriding (Metallurgiya, Moscow, 1991).

25. K. Hauffe, Reactions in and on Solids (Springer-Verlag, Berlin, 1955).

26. S. D. Hertsriken and I. Ya. Dekhtyar, Diffusion in Metals and Alloys in Solid Phase (Gosizdat Fiz.-Mat. Lit., Moscow, 1960).

27. S. Torizuka, A. Ohmori, S. V. S. N. Murty, and K. Nagai, "High Z-Large Strain Deformation Processing and Its Applications," Mater. Sci. Forum. 503504, 329-334 (2006).

28. J.-H. Hang and S. Torizuka, "Dynamic Recrystallization by Large Strain Deformation with a High Strain Rate in an Ultralow Carbon Steel," Scripta Mater. 57, 1048-1051 (2007).

SPELL: 1. ok 